\title{
Editorial
}

\section{Super-Resolution Imaging: Analysis, Algorithms, and Applications}

\author{
Michael Ng, ${ }^{1}$ Tony Chan, ${ }^{2}$ Moon Gi Kang, ${ }^{3}$ and Peyman Milanfar ${ }^{4}$ \\ ${ }^{1}$ Department of Mathematics, Hong Kong Baptist University, Kowloon Tong, Hong Kong \\ ${ }^{2}$ Department of Mathematics, University of California, Los Angeles, CA 90095-1555, USA \\ ${ }^{3}$ Department of Electrical and Electronic Engineering, Yonsei University, Seoul 120-749, Korea \\ ${ }^{4}$ Department of Electrical Engineering, University of California, Santa Cruz, CA 95064, USA
}

Received 2 August 2005; Accepted 2 August 2005

Copyright (c) 2006 Michael Ng et al. This is an open access article distributed under the Creative Commons Attribution License, which permits unrestricted use, distribution, and reproduction in any medium, provided the original work is properly cited.

The recent increase in the widespread use of digital imaging technologies in consumer (e.g., digital video) and other markets (e.g., security and military) has brought with it a simultaneous demand for higher-resolution (HR) images. The demand for such images can be partially met by algorithmic advances in super-resolution (SR) technology in addition to hardware development. Such HR images not only give the viewer a more pleasing picture but also offer additional details that are important for subsequent analysis in many applications.

The current hardware approach to obtain HR images mainly relies on sensor manufacturing technology that attempts to increase the number of pixels per unit area by reducing the pixel size. However, the cost for high-precision optics and sensors may be prohibitive for general purpose commercial applications, and there is a limitation to pixel size reduction due to shot noise encountered in the sensor itself. Therefore, a resolution enhancement (SR) approach using computational, mathematical, and statistical techniques has received a great deal of attention recently. The relevant signal processing technology for this SR approach to highquality imaging is the topic of this special issue. The scope of techniques intended to overcome the above limitations that will be covered in this special issue will include enhancement in spatial resolution for both gray-scale and color images and video, suppression of signal-dependent noise, and various other associated artifacts.

Because of the recent emergence of many key-relevant computational, mathematical, and statistical techniques, and the increasing importance of digital imaging technology, a special issue of the EURASIP JASP dedicated to the topic of SR imaging is quite timely.
This special issue contains sixteen articles. The first seven articles by M. Vega et al., M.-C. Pan, S. Farsiu et al., G. M. Callico et al., B.-W. Jeon et al., N. K. Bose et al., and T. Q. Pham et al. are on the computational, mathematical and statistical techniques for SR imaging. The next three articles by P. Vandewalle et al., M. Trimeche et al., and M. Balci and H. Foroosh are on the subject of subpixel registration of lowresolution images in image reconstruction. The next four articles by C. V. Jiji and S. Chaudhuri, S. Rajaram et al., F. Humblot and A. Mohammad-Djafari, and T. A. Stephenson and T. Chen are on applying different learning techniques in the SR image reconstruction. The last part with two articles by S. Zhang and $\mathrm{X}$. Li is about the application of SR reconstruction techniques in optical systems.

The Guest Editors thank all the authors who have contributed to this special issue. Special thanks are also due to the reviewers for their constructive suggestions and comments following their evaluation of the articles. The Guest Editors are indebted to the Editorial Board of EURASIP JASP for providing this opportunity to edit this special issue.
Michael $\mathrm{Ng}$

Tony Chan

Moon Gi Kang

Peyman Milanfar 
Michael Ng is a Professor at the Mathematics Department, Hong Kong Baptist University, and is an Honorary Professor in the Department of Mathematics, and an Adjunct Research Fellow in the E-Business Technology Institute at the University of Hong Kong. He was one of the finalists and honourable mention of Householder Award IX, in 1996, at Switzerland, and he obtained an excellent young researcher's presentation at

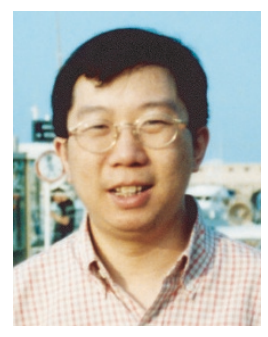
Nanjing International Conference on Optimization and Numerical Algebra, 1999. In 2001, he was selected as one of the recipients of the Outstanding Young Researcher Award of the University of Hong Kong. He has published and edited several books, and published extensively in international journals and conferences, and has organized and served in many international conferences. Now he serves on the Editorial Boards of SIAM Journal on Scientific Computing, Numerical Linear Algebra with Applications, International Journal of Data Mining and Bioinformatics, Multidimensional Systems and Signal Processing, International Journal of Computational Science and Engineering, Numerical Mathematics: A journal of Chinese Universities (English Series), and several special issues of international journals.

Tony Chan has his scientific background in mathematics, computer science, and engineering. He received his B.S. and M.S. degrees from California Institute of Technology and his Ph.D. degree from Stanford University, and taught at Yale University before joining the UCLA faculty in 1986. He became the Chair of the Department of Mathematics in 1997. He was one of the principal investigators who made the suc-

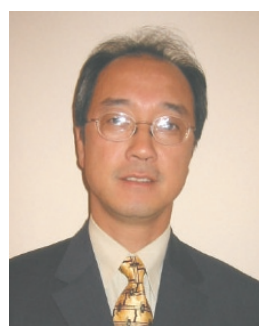
cessful proposal to NSF to form the Institute for Pure and Applied Mathematics at UCLA, with a vision to promote collaborations between the mathematical sciences with the general scientific and engineering disciplines. He served as an IPAM's Director from 2000 to 2001. Since July 2001, he has been the Dean of Physical Sciences Division at UCLA. His current research interests include mathematical image processing and computer vision, VLSI physical design, and human brain mapping. He is an active Member of many scientific societies, including SIAM, AMS, and IEEE.

Moon Gi Kang received his B.S. and M.S. degrees in electronics engineering from Seoul National University, Korea, in 1986 and 1988, respectively, and his Ph.D. degree in electrical engineering from Northwestern University in 1994. He was an Assistant Professor at the University of Minnesota, Duluth, from 1994 to 1997, and since 1997 he has been in the Department of Electrical and Electronic Engineering, Yonsei Uni-

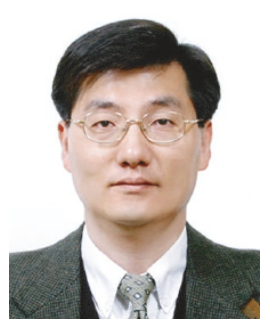
versity, Seoul, Korea, where he is currently a Professor. His current research interests include image and video filtering, restoration, enhancement, and reconstruction. He served and currently serves as the Editorial Board Member for the IEEE Signal Processing Magazine, the Editor of SPIE Milestone Series Volume (CCD and CMOS imagers), the Guest Editor of the IEEE SPM Special Issue on Superresolution Image Reconstruction (May, 2003), the Editor of EURASIP Journal on Applied Signal Processing, and the Reviewer for the IEEE Transactions on Image Processing. He has also served as the Associate Editor for the Journal of Broadcast Engineering and Journal of IEEK (the Institute of Electronics Engineers of Korea). He is the recipient of the 2002 HaeDong Foundation Best Paper Award and the recipient of the 2000 Award of Teaching Excellence from the School of Electrical and Electronic Engineering at Yonsei University.

Peyman Milanfar received the B.S. degree in electrical engineering and mathematics from the University of California, Berkeley, in 1988, and the S.M., E.E., and Ph.D. degrees in electrical engineering from the Massachusetts Institute of Technology, in 1990, 1992, and 1993, respectively. Until 1999, he was a Senior Research Engineer at SRI International, Menlo Park, Calif. He is currently an Associate Professor of electri-

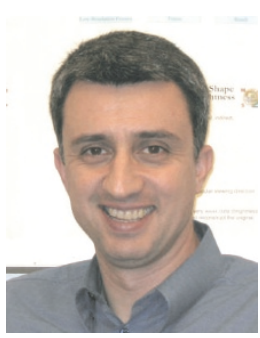
cal engineering at the University of California, Santa Cruz. He was a Consulting Assistant Professor of computer science at Stanford University from 1998 to 2000, and a Visiting Associate Professor there in 2002. His technical interests are in statistical signal and image processing, and inverse problems. He won a National Science Foundation CAREER Award in 2000, was an Associate Editor for the IEEE Signal Processing Letters from 1998 to 2001, and is a Senior Member of the IEEE. 